\title{
Wireless Social Community Networks: A Game-Theoretic Analysis
}

\author{
Mohammad Hossein Manshaei*, Julien Freudiger*, Mark Félegyházi*, Peter Marbach ${ }^{\dagger}$, and Jean-Pierre Hubaux* \\ * Laboratory for computer Communications and Applications (LCA), EPFL, Lausanne, Switzerland \\ Email:\{hossein.manshaei, julien.freudiger, mark.felegyhazi, jean-pierre.hubaux\}@epfl.ch \\ ${ }^{\dagger}$ Department of Computer Science, University of Toronto, Canada \\ Email: marbach@cs.toronto.edu
}

\begin{abstract}
Wireless social community networks formed by users with a WiFi access point have been created as an alternative to traditional wireless networks that operate in the licensed spectrum. By relying on access points owned by users for access, wireless community networks provide a wireless infrastructure in an inexpensive way. However, the coverage of such a network is limited by the set of users who open their access points to the social community. Currently, it is not clear to what degree this paradigm can serve as a replacement, or a complimentary service, of existing centralized networks operating in licensed bands. In this paper, we study the dynamics of wireless social community networks using, as well as the situation where a wireless social community networks co-exists with a traditional wireless network operating in the licensed spectrum.
\end{abstract}

\section{INTRODUCTION}

Wireless social community networks have been created as an alternative to wireless networks that operate in the licensed spectrum for providing wireless data access in urban areas. Traditionally, wireless networks have been operated using licensed spectrum and have been operated by a central authority. Such networks can guarantee a high quality of service $(\mathrm{QoS})$ in terms of network coverage; however this comes at the expense of substantial deployment and maintenance costs. Wireless social community networks operate in the unlicensed spectrum and rely on users having a WiFi access point to provide access. Thus, there is no need for an operator to make substantial initial investment to buy the spectrum license. Furthermore, the access points (AP) are inexpensive, easy to deploy and maintain. However, wireless social community might have a poor coverage as the coverage depends on number of users that subscribe to the social community network.

In this paper, we study how effective wireless social communities networks are. We are in particular interested in the case where a wireless social community networks competes with traditional a licensed band network. To do this, we first investigate how users decide whether or not to join the social community network, and study the evolution of the number of members in the community by modeling users' payoffs as a function of the subscription fee $^{1}$, as well as the operators' provided coverage. Next, we study the competition between a social community operator and a wireless network operating in the licensed spectrum using a game-theoretic framework.

${ }^{1}$ Note that the subscription fee corresponds to the price users have to pay. Hence, we use the two terms interchangeably in the paper.
For the resulting non-cooperative game, we investigate the existence a Nash equilibrium, and characterize the number of users that subscribe to each service provider under a Nash equilibrium. Due to space constraints we present our results without proof and will focus at several instances on particular special cases, as discussed in the following.

\section{System MODEL}

Consider two network operators, a traditional licensed band operator (LBO or $\ell$ ) and social community operators (SCO or $s$ ), that compete for providing access in a given area to a set of $N$ users. Each provider charges a subscription fee $P_{i}, i \in$ $\{l, s\}$. Users decided at discrete time instances $t=1,2, \ldots$ to which provider they subscribe. If a user subscribes to provider $i, i \in\{l, s\}$, then the user pays a subscription fee $P_{i}[t]$ at time $t$. Let $n_{s}[t]$ be the fraction of users that subscribes to the SCO, and let $n_{l}[t]$ be the fraction for users subscribing to the LBO.

Let $Q_{l}$ and $Q_{s}$ be the network coverage that the LBO and $\mathrm{SCO}$, respectively, provide. In the following we assume that the LBO always provides full coverage and all users that subscribe to the LBO always have access to a base station, i.e we have that $Q_{l}[t]=1, t \geq 0$. On the other hand, the coverage $Q_{s}[t]$ provided by the SCO at time $t$ depends on the number of users $n_{s}[t]$ that subscribe to the SCO. Here, we use the following simple relation to model this situation: we let

$$
Q_{s}[t]=\min \left\{1, \lambda n_{s}[t]\right\}
$$

where $\lambda>0$ is a positive constant modeling the density of the access points owned by users (see also Fig. 1). For example, a large $\lambda$ captures the case where access points are very dense as it might be the case in a city center. Given the subscription

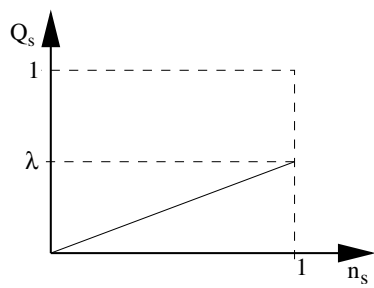

(a)

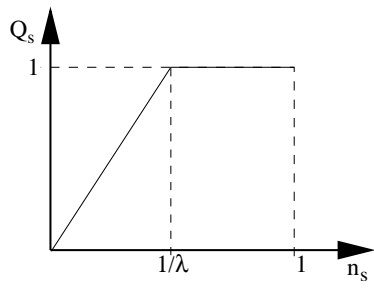

(b)
Fig. 1. Relation between fraction of subscribers $n_{s}$ and the social community coverage $Q_{s}$ : (a) $0<\lambda \leq 1$, (b) $1<\lambda$. 
fee $P_{i}$ and coverage $Q_{i}$ of a provider $i \in\{l, s\}$, the utility that a given user $v$ obtains by subscribing to provider $i$ is given by

$$
u_{v}^{i}=a_{v} Q_{i}-P_{i}
$$

where $a_{v}$ is a non-negative parameter that characterizes the user sensitivity with respect to coverage. User $v$ only considers subscribing to provider $i$ if the resulting utility $u_{v}^{i}$ is nonnegative.

In the following we assume a large user population and that the users' sensitivity towards coverage is uniformly distributed in $[\alpha, \beta], \alpha \geq 0$. As a result, we let the fraction of users with a sensitivity towards coverage that is larger than a given value $x \in[\alpha, \beta]$ be given by $\frac{\beta-x}{\beta-\alpha}$.

The payoff of operator $i, i \in\{\ell, s\}$, at time $t$ is given by

$$
u_{i}[t]=N \cdot n_{i}[t] \cdot P_{i}-c_{i}, \quad i \in\{\ell, s\},
$$

where $N n_{i}[t]$ is the total number of users subscribing to provider $i$ at time $t$ and $c_{i}$ is the operating cost of provider $i$ per unit time slot.

\section{RELATED WORK}

The wireless community networks over unlicensed band have been recently deployed by some ISPs such as Free [4] in France or FON, a worldwide WiFi community operator funded by Google and Skype [3]. A charging model for wireless social community networks without a centralized authority is proposed by Efstathiou et al. [5]. Their solution relies on reciprocity among subscribers. In [6], Zemlianov and de Veciana evaluate using a stochastic geometric model, the cooperation between licensed band WAN and WLAN service providers. A complete evaluation of our model for $\lambda=1$ is presented in [7].

\section{Optimal Pricing StRategy of LBO}

Consider the situation where the LBO is the only wireless access provider in a given area. For this case we are interested in determining the optimal price $P_{l}^{*}$, that the LBO should charge per unit time in order to maximize its revenue. Note that under a given price $P_{l}$, only user for which the payoff $u_{v}^{l}$ given by Eq. (1) is non-negative subscribes to the LBO, and the fraction of users $n_{l}$ that subscribes to the LBO under the price $P_{l}$ is given by

$$
n_{\ell}=\frac{1}{\beta-\alpha}\left(\beta-\max \left\{\alpha, P_{\ell}\right\}\right) .
$$

The resulting payoff of the LBO is given by

$$
u_{\ell}=\frac{N}{\beta-\alpha}\left(\beta-\max \left\{\alpha, P_{\ell}\right\}\right) \cdot P_{\ell}-c_{\ell}
$$

The following lemma shows the optimal price of LBO.

Lemma 1: The optimal price $P_{l}^{*}$ is given by

$$
P_{\ell}^{o p t}=\max \left\{\alpha, \frac{\beta}{2}\right\}
$$

The fraction of users $n_{l}^{*}$ that subscribes to the LBO under the price $P_{l}^{*}$ is given by

$$
n_{l}^{*}=\max \left\{1, \frac{1}{2} \frac{\beta}{\beta-\alpha}\right\} .
$$

\section{Optimal Pricing Strategy of SCO}

Next we consider the situation where the SBO is the only wireless provider in a given area. For this case, we are again interested in determining the optimal price $P_{s}^{*}$ the SCO should charge per unit time in order to maximize its revenue. Here we assume that at time $t$ users observe the coverage $Q_{s}(t-1)$ at time $t-1$ and the subscription fee $P_{s}[t]$ at time $t$. Using this information, a user $v$ then subscribes to the SCO if

$$
u_{v}^{s}[t]=a_{v} Q_{s}(t-1)-P_{s}[t] \geq 0 .
$$

Under a fixed price $P_{s}[t]=P_{s}, t \geq 0$, the fraction of users $n_{s}[t]$ that subscribe to the SCO at time $t$, and hence the coverage $Q_{s}[t]$ of the SCO at time $t$, is then a function of the coverage $Q_{s}(t-1)$ at the previous time step. In particular, we have that

$$
Q_{s}[t]=\min \left\{1, \frac{\lambda}{\beta-\alpha}\left(\beta-\max \left\{\alpha, \frac{P_{s}}{Q_{s}[t-1]}\right\}\right)\right\}
$$

In the following, we study (a) how the coverage $Q_{s}[t]$ evolves over time under a fixed price $P_{s}$ and (b) what price $P_{s}^{*}$ the SCO should charge in order to maximize its revenue. For this analysis, we focus on the case where $\lambda \in(0,2)$ and $\beta \geq \frac{2}{2-\lambda} \alpha$. The analysis, and system behavior, for the general case is similar to this situation. Before we start our analysis, we observe the following results.

Lemma 2: If $Q_{s}[0]=0$ and $P_{s}[t]=P_{s}>0, t \geq 0$, then we have that $Q_{s}[t]=0, t \geq 0$.

Lemma 3: If $P_{s}[t]=P_{s}>0, t \geq 0$, and $\frac{P_{s}}{Q_{s}[0]} \leq \alpha$ then $Q_{s}[t]=\min \{1, \lambda\}, t \geq 0$.

\section{A. Dynamics of the SCO under a Fixed Price $P_{s}$}

In this subsection we assume that the SCO charges a fixed price $P_{s}$ and study for this case how the coverage $Q_{s}[t]$ evolves as a function of the initial coverage $Q_{s}[0]$.

Let $Q_{s, 1}$ and $Q_{s, 2}$ be given as follows,

$$
Q_{s, 1}=\frac{\beta \lambda-\sqrt{\beta^{2} \lambda^{2}-4(\beta-\alpha) P_{s} \lambda}}{2(\beta-\alpha)}
$$

and

$$
Q_{s, 2}=\frac{\beta \lambda+\sqrt{\beta^{2} \lambda^{2}-4(\beta-\alpha) P_{s} \lambda}}{2(\beta-\alpha)} .
$$

The following results characterize the dynamics of the coverage $Q_{s}[t]$ for the above case. In our analysis, we distinguish different cases depending on the price $P_{s}$ (see also Fig. 2). We first consider the case where the price $P_{s}$ is low.

Lemma 4: Suppose that

$$
P_{s} \in\left[0, \beta-\frac{1}{\lambda}(\beta-\alpha)\right]
$$


If $Q_{s}[0]<Q_{s, 1}$ then $\lim _{t \rightarrow \infty} Q_{s}[t]=0$; otherwise $\lim _{t \rightarrow \infty} Q_{s}[t]=\min \{1, \lambda\}$.

The next result is for the case where the price $P_{s}$ is higher than $\beta-\frac{1}{\lambda}(\beta-\alpha)$ but smaller than $\frac{\beta^{2} \lambda}{4(\beta-\alpha)}$.

Lemma 5: Suppose that

$$
\beta-\frac{1}{\lambda}(\beta-\alpha)<P_{s}<\frac{\beta^{2} \lambda}{4(\beta-\alpha)} .
$$

If $Q_{s}[0]<Q_{s, 1}$ then we have that $\lim _{t \rightarrow \infty} Q_{s}[t]=0$. If $Q_{s}[0]>Q_{s, 1}$ then $\lim _{t \rightarrow \infty} Q_{s}[t]=Q_{s, 2}$. Finally, if $Q_{s}[0]=$ $Q_{s, 1}$ then we have $Q_{s}[t]=Q_{s, 1}, t \geq 0$.

The third case is the situation where the price $P_{s}$ is exactly equal to $\frac{\beta^{2} \lambda}{4(\beta-\alpha)}$.

Lemma 6: Suppose that

$$
P_{s}=\frac{\beta^{2} \lambda}{4(\beta-\alpha)} .
$$

If $Q_{s}[0]<Q_{s, 1}$ then $\lim _{t \rightarrow \infty} Q_{s}[t]=0$. If $Q_{s}[0] \geq Q_{s, 1}$ then $\lim _{t \rightarrow \infty} Q_{s}[t]=Q_{s, 1}=Q_{s, 2}=\frac{\beta \lambda}{2(\beta-\alpha)}$.

Finally, we consider the case where $P_{s}$ is high, i.e. if $P_{s}$ is larger than $\frac{\beta^{2} \lambda}{4(\beta-\alpha)}$.

Lemma 7: If

$$
P_{s}>\frac{\beta^{2} \lambda}{4(\beta-\alpha)}
$$

then we have that $\lim _{t \rightarrow \infty} Q_{s}[t]=0$.

(a)

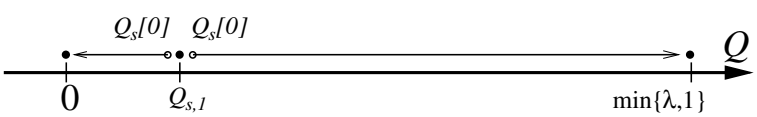

(b)

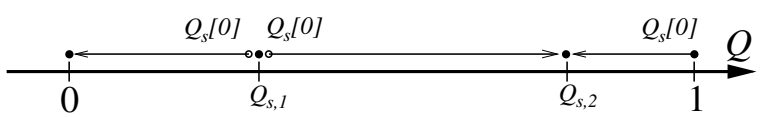

(c)

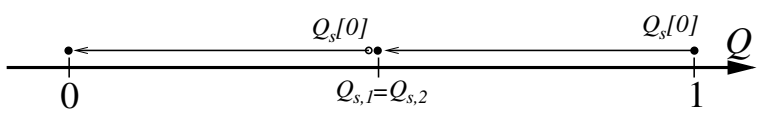

(d)

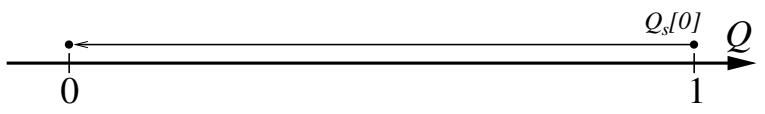

Fig. 2. Dynamics of SCO for $\lambda<2$ and $\beta \geq \frac{2}{2-\lambda} \alpha$ : (a) $0<P_{s} \leq$ $\beta-\frac{1}{\lambda}(\beta-\alpha)$, (b) $\beta-\frac{1}{\lambda}(\beta-\alpha)<P_{s}<\frac{\beta^{2} \lambda}{4(\beta-\alpha)}$, (c) $P_{s}=\frac{\beta^{2} \lambda}{4(\beta-\alpha)}$, (d) $P_{s}>\frac{\beta^{2} \lambda}{4(\beta-\alpha)}$.

The above results imply that given the initial coverage $\left.Q_{[} 0\right]$ and a fixed subscription fee $P_{s}$, the fraction of users that subscribe to the social community network converges to a well-defined limit point: let $n_{s}\left(Q_{s}[0], P_{s}\right)$ denote this limit point.

\section{B. Optimal Static Price}

Let $n_{s}\left(Q_{s}[0], P_{s}\right)$ the fraction of users that subscribe to the $\mathrm{SCO}$ at the limit point corresponding to a given value of $\left.Q_{[} 0\right]$ and $P_{s}$, and let

$$
u_{s}\left(Q_{s}[0], P_{s}\right)=N n_{s}\left(Q_{s}[0], P_{s}\right) P_{s}-c_{i}
$$

be the corresponding payoff. In this section, we characterize the optimal price $P_{s}^{*}$ that the SCO should charge in order to maximize the payoff $u_{s}\left(Q_{s}[0], P_{s}\right)$ for a given initial coverage $Q_{s}[0]$, i.e. we have

$$
P_{s}^{*}=\arg \max _{P_{s} \geq 0} u_{s}\left(Q_{s}[0], P_{s}\right)
$$

If the initial coverage $Q_{s}[0]$ is small, then we obtain the following result.

Theorem 1: If we have that $Q_{s}[0] \leq \lambda \frac{\beta}{\beta-\alpha}-1$, then

$$
P_{s}^{*}=Q_{s}[0] \cdot\left(\beta-\frac{1}{\lambda}(\beta-\alpha) \cdot Q_{s}[0]\right)
$$

and $n_{s}\left(Q_{s}[0], P_{s}^{*}\right)=1$.

Under $Q_{s}[0]$ and $P_{s}^{*}$ in Theorem 1 , the dynamics of the social community is as given by Lemma 4 (see also Fig. 2 (a)). Note that the optimal price $P_{s}^{*}$ in Theorem 1 depends on the initial coverage; the intuition behind this is that when the initial coverage $Q_{s}[0]$ is small, then the SCO needs to choose a subscription fee small enough in order to prevent the coverage $Q_{s}[t]$ to converge to 0 .

If the initial coverage is large, then we have the following result.

Theorem 2: If $Q_{s}[0]>\lambda \frac{\beta}{\beta-\alpha}-1$ and $\beta<\frac{3}{\lambda} \alpha$, then

$$
P_{s}^{*}=\beta-\frac{1}{\lambda}(\beta-\alpha)
$$

and $n_{s}\left(Q_{s}[0], P_{s}^{*}\right)=1$.

If $Q_{s}[0]>\lambda_{\beta-\alpha}-1$ and $\beta \geq \frac{3}{\lambda} \alpha$, then

$$
P_{s}^{*}=\frac{2 \lambda}{9} \frac{\beta^{2}}{(\beta-\alpha)}
$$

and $n_{s}\left(Q_{s}[0], P_{s}^{*}\right)=\frac{2}{3} \frac{\beta}{\beta-\alpha}$.

Note that the optimal price $P_{s}^{*}$ given in Theorem 2 depends on $\alpha, \beta$, and $\lambda$, but not on the initial coverage $Q_{s}[0]$. If $\beta<\frac{3}{\lambda} \alpha$, then under $Q_{s}[0]$ and $P_{s}^{*}$ as in Theorem 1 the dynamics of the social community is as given by Lemma 4 (see also Fig. 2 (a)). If $\beta<\frac{3}{\lambda} \alpha$, then under $Q_{s}[0]$ and $P_{s}^{*}$ as in Theorem 1 the dynamics of the social community is as given by Lemma 5 (see also Fig. 2 (b))

\section{Optimal Dynamic Pricing Strategy}

So far we assumed that the subscription fee that the SCO charges is fixed. Now suppose that the SCO can adjust the subscription fee over time: in this case, how should the SCO adapt its price in order to converge to an operating point that maximizes its payoff? One can show that this following pricing strategies achieve this.

If $\beta<\frac{3}{\lambda} \alpha$, then set the initial price $P_{s}[0]=0$ and afterwards charge

$$
P_{s}[t]=\beta-\frac{1}{\lambda}(\beta-\alpha), \quad t \geq 0 .
$$

If $\beta \geq \frac{3}{\lambda} \alpha$, then set the initial price $P_{s}[0]=0$ and afterword charge

$$
P_{s}[t]=\frac{2 \lambda}{9} \frac{\beta^{2}}{(\beta-\alpha)}, \quad t \geq 0
$$


The intuition behind the above pricing strategies is that setting the price $P_{s}[0]$ equal to 0 will lead to $n_{s}[1]=1$ and to a large coverage at time 1 . Once a large coverage has been achieved, then the results in Theorem 2 can be used to determine the optimal subscription fee.

\section{Competition Between a SCO AND A LBO}

Next we consider the situation where a LBO and SCO coexist in a given area, and compete for mobile users to subscribe to their service. We model this situation as a non-cooperative pricing game where two operators are the players [1]. The operators compete through their subscription price and the the strategy of operator $i$ in the game is given by its price $P_{i}$.

Again we assume that users make decision at discrete time steps $t=1,2, \cdots$. Recall the definition of the utility $u_{v}^{i}$ that user $v$ achieves when it subscribes to a provider $i \in\{l, s\}$. Given subscription fees $P_{l}$ and $P_{s}$, and observing the coverage $Q_{s}[t-1]$ of the SCO at time $t-1$, user $v$ will choose at time $t$ the provider $i$ which leads to the largest utility $u_{v}^{i}$ at time $t$. Of course, user $v$ will only subscribe to this provider if the resulting utility is non-negative; otherwise the user will not subscribe to any provider. Let $n_{l}[t]$ and $n_{s}[t]$ the resulting fraction of users that subscribe to the LBO and the SCO, respectively, at time $t$.

Given fixed prices $P_{l}$ and $P_{s}$, we call $Q_{s}\left(P_{l}, P_{s}\right)$ an equilibrium coverage if for $Q_{s}[0]=Q_{s}\left(P_{l}, P_{s}\right)$ we have under $P_{l}$ and $P_{s}$ that $Q_{s}[t]=Q_{s}\left(P_{l}, P_{s}\right), t \geq 0$. Similarly, we define the equilibrium markets shares $n_{l}\left(P_{l}, P_{s}\right)$ and $n_{s}\left(P_{l}, P_{s}\right)$, and the corresponding equilibrium payoffs $u_{l}\left(P_{l}, P_{s}\right)$ and $u_{s}\left(P_{s}, P_{l}\right)$ of the LBO and the SCO, respectively. Using the above definitions, a Nash equilibrium for the above game is given as follows.

Definition 1: The price pair $\left(P_{l}^{*}, P_{s}^{*}\right)$ constitutes a Nash equilibrium if for each operator $i \in\{l, s\}$ we have

$$
u_{i}\left(P_{i}^{*}, P_{j}^{*}\right) \geq u_{i}\left(P_{i}, P_{j}^{*}\right), \quad \forall P_{i} \geq 0 .
$$

At a Nash equilibrium, none of the operators has an incentive to unilaterally change its subscription fee as this will not increase its payoff. In the following we study whether there exists a Nash equilibrium for the above game. To simplify the analysis, we assume that $\alpha=0$.

Theorem 3: Suppose that $\alpha=0$. If $\lambda \in(0,3)$ then there exists a unique Nash equilibrium given by

$$
\left(P_{\ell}^{*}, P_{s}^{*}\right)=\left(\frac{\beta}{2} \cdot \frac{1-Q_{s}^{*}}{1-\frac{Q_{s}^{*}}{4}}, \frac{\beta Q_{s}^{*}}{4} \cdot \frac{1-Q_{s}^{*}}{1-\frac{Q_{s}^{*}}{4}}\right)
$$

where $Q_{s}^{*}=2-\sqrt{4-\lambda}$. The fraction of users that subscribe to the SCO at the Nash equilibrium is given by $n_{s}^{*}=\frac{1}{\lambda} Q_{s}^{*}=$ $\frac{1}{2+\sqrt{4-\lambda}}$, and the fraction of users that subscribe to the LBO is given by $n_{l}^{*}=\frac{2}{2+\sqrt{4-\lambda}}$.

If $\lambda \geq 3$, then there exists a unique Nash equilibrium $\left(P_{l}^{*}=0, P_{s}^{*}=0\right)$ with $Q_{s}\left(P_{l}^{*}, P_{s}^{*}\right)=1$. However the fraction of users that subscribe the each operator are not uniquely determined. In particular, any market share $n_{l}^{*}$ and $n_{s}^{*}$ such that $n_{s}^{*} \geq 1 / \lambda$ and $n_{l}^{*}+n_{s}^{*}=1$ may be realized at a Nash equilibrium.

The above analysis implies that there always exists a unique Nash equilibrium. For $\lambda \in(0,3)$ the market share of each provider is uniquely determined. Furthermore, for $\lambda \in(0,3)$, we have that (a) the market share (i.e. the fraction of users that subscribe to an operator) of both the LBO and SCO increase as $\lambda$ increases and (b) the market share of the LBO is always twice as large as the market share of the SCO.

The subscription fees $\left(P_{l}^{*}, P_{s}^{*}\right)$ charged at a Nash equilibrium tend to decrease as $\lambda$ increases. This suggests that the SCO influences the pricing behavior of a LBO, and that the presence of a SCO in area with a dense network of WiFi access points might be able to significantly reduce the fees charged for wireless access.

\section{CONCLUSIONS}

In this paper we have analyzed the competition between a social community and licensed band operators using a gametheoretic approach. We showed that there always exists a unique a Nash equilibrium for the resulting non-cooperative game, and characterized the number of users that subscribe to each service provider under a Nash equilibrium.

The results that we obtained are intuitive, suggesting that the simple model that we used for our analysis is indeed able to capture the main features of the competition between a LBO and SCO. As expected, the results suggest that a SCO tends to successful in areas with a high density of access points owned by users.

Possible future work is to consider more general distribution for the users' sensitivity towards coverage, as well as to take interference effects into account that might occur when too many users subscribe to the SCO.

\section{REFERENCES}

[1] M. J. Osborne and A. Rubinstein, "A Course in Game Theory," The MIT Press, Cambridge, MA, 1994.

[2] J. Nash, "Non-Cooperative Games," The Annals of Mathematics, Vol. 54 No.2, PP 286-295, 1951.

[3] "www.fon.com"

[4] "www.free.fr"

[5] E. C. Efstathiou, P. A. Frangoudis, and G. C. Polyzos. Stimulating participation in wireless community networks. In INFOCOM, 2006.

[6] A. Zemlianov and G. de Veciana. Cooperation and decision making in wireless multiprovider setting. In INFOCOM, 2005.

[7] M.H. Manshaei, J. Freudiger, M. Felegyhazi, P. Marbach, J.-P. Hubaux, "On Wireless Social Community Networks," INFOCOM, 2008. 